\title{
Treatment of EEG Abnormalities in Benign Childhood Epilepsy with Centrotemporal Spikes
}

\author{
Hisako Yamamoto ${ }^{1,2}$, Kanako Takeda ${ }^{1,2}$, Yusaku Miyamoto ${ }^{1,2}$, and Hitoshi Yamamoto ${ }^{2}$
}

(Received for Publication: November 22, 2019)

\begin{abstract}
Benign childhood epilepsy with centrotemporal spikes (BECTS) is the most common childhood type of epilepsy. Whether treatment should be initiated and the necessity of treatment for abnormality of interictal encephalography (EEG) in BECTS remain controversial. We investigated the relationship between age in months at seizure onset, duration of interictal EEG abnormality, and the period until the disappearance of seizures in BECTS patients receiving in treatment (TR group) or no-treatment (NT group). There was a negative correlation between the age at seizure onset and the duration of interictal EEG abnormality $(p<0.01)$, whereas there was a positive correlation between the duration of interictal EEG abnormality and the period until the disappearance of seizures $(\mathrm{p}<0.05)$. There was a significant difference in the duration of interictal EEG abnormalities and the period until the disappearance of seizures between the NT and TR groups $(p<0.01)$. Our results suggested that there may be an association between interictal EEG abnormalities and the duration of seizure in BECTS, indicating that interictal EEG abnormality may be a target for treatment.
\end{abstract}

\section{Key words}

Benign childhood epilepsy with centrotemporal spikes (BECTS), epilepsy treatment

\section{Introduction}

Benign childhood epilepsy with centrotemporal spikes (BECTS) is one of the most common childhood epilepsy syndromes, with a prevalence of 1525\%. ${ }^{1)}$ Although seizures usually occur in children aged 3-13 years, the prognosis is favorable and the condition typically resolves by puberty. BECTS is characterized by focal seizures that typically occur only at the extremities, or as clonic or tonic seizures of the oral and facial region. Seizures may frequently become secondarily generalized. The seizures frequency is low, typically $2-5$ seizures in total.

Considering the side effects of antiepileptic drugs (AEDs) and given that BECTS has a low frequency of seizures and a high probability of spontaneous remission before adulthood, follow-up observation without treatment has been acceptable in many cases. However, the response to drug treatment is typ- ically favorable. In a previous study, treatments focused on seizures, rather than abnormality on electroencephalography (EEG), were applied to two-thirds of the BECTS cases, with the non-treated cases accounting for the remaining one-third of cases. ${ }^{2)}$ Even reports that oppose treatment agree that therapy should be performed in those who experience repetitive seizures or generalized tonic-clonic seizures.

In contrast, it has recently been reported that interictal EEG abnormalities are associated with cognitive dysfunction and neuropsychiatric disorders. ${ }^{3)}$ However, the necessity of treatment in patients with BECTS who show EEG abnormalities is controversial. When changes in EEG are observed in accordance with the spontaneous course of BECTS, it has been reported that centrotemporal spikes disappear 6 months after diagnosis in $30 \%$ of cases. ${ }^{4)}$ Moreover, it was reported that treatment with AEDs did not shorten the persistence of the EEG abnormalities."

1 Division of Pediatrics, Kawasaki Municipal Tama Hospital, St. Marianna University School of Medicine

2 Department of Pediatrics, St. Marianna University School of Medicine 
Thus, it is controversial whether intervention should be initiated in BECTS patients and whether treatment for interictal EEG abnormalities is necessary. In the present study, we investigated whether there were differences in the durations of seizures and EEG abnormalities between treated and non-treated BECTS patients.

\section{Materials and Methods}

Subjects in this retrospective study included children who visited our hospital with a main complaint of seizures between February 2006 and October 2018 and who were diagnosed as having BECTS based on clinical symptoms and EEG findings obtained by two pediatric neurologists and epilepsy physicians. Age, sex, follow-up period, outcome, nature of the seizure(s), family history, medical history of fever seizures, presence or absence of consanguineous marriage, and the EEG findings were obtained from medical records. Interictal EEG was performed based on the international 10-20 system during sleep (Comet EEG System, Grass Technologies, West Warwick, RI, USA). EEG abnormality was defined as centrotemporal spikes or epileptic discharges.

In addition, in subjects followed-up for $\geq 1$ year and who underwent EEG $\geq 2$ times, according to the report by Kim et al., ${ }^{5)}$ Spearman's ranked correlation coefficient test was performed to determine the relationship of age in months at the initial seizure, the duration of EEG abnormality, and the period until the disappearance of seizures. The intervals between the performance of EEG tests were at least six months. The subjects were divided into the non-treated (NT) group and treated (TR) group.

The study protocol was reviewed and approved by the institutional review boards of the participating institutions (approval no.: 4262).

\section{Statistical analysis}

The Mann-Whitney test was performed for age in months at seizure onset, the duration of interictal electroencephalographic abnormality, the period until the disappearance of seizures, and follow-up duration. Fisher's exact test was used to compare the sex ratio, medical history of febrile seizures, and the presence or absence of complications due to developmental disorders, the focus of the EEG, and generalization or not on EEG between the NT and TR groups. Statistical analyses were performed using jsSTAR version 9.7.0j. Odds ratios and 95\% confidence intervals (CIs) were calculated with HAD version 16.00. A p-value of $<0.05$ was considered to indicate statistical significance.

\section{Results}

\section{Clinical features}

The characteristics of the patients are given in Figure 1 and Table 1. Twenty-nine pediatric patients (16 [55\%] males, 13 [45\%] females) were included in the present study. During the follow-up period, 10 [34\%] children had completed treatment, 13 [45\%] were on treatment, 4 [14\%] had discontinued the treatment by themselves, and 2 [7\%] had changed hospitals for treatment. At the initial, seizure patients were, on average, about 7.5 years old, and the mean age at the final seizure was about 8.6 years. Seven [24\%] patients were followed-up for $\geq 2$ years to $<3$

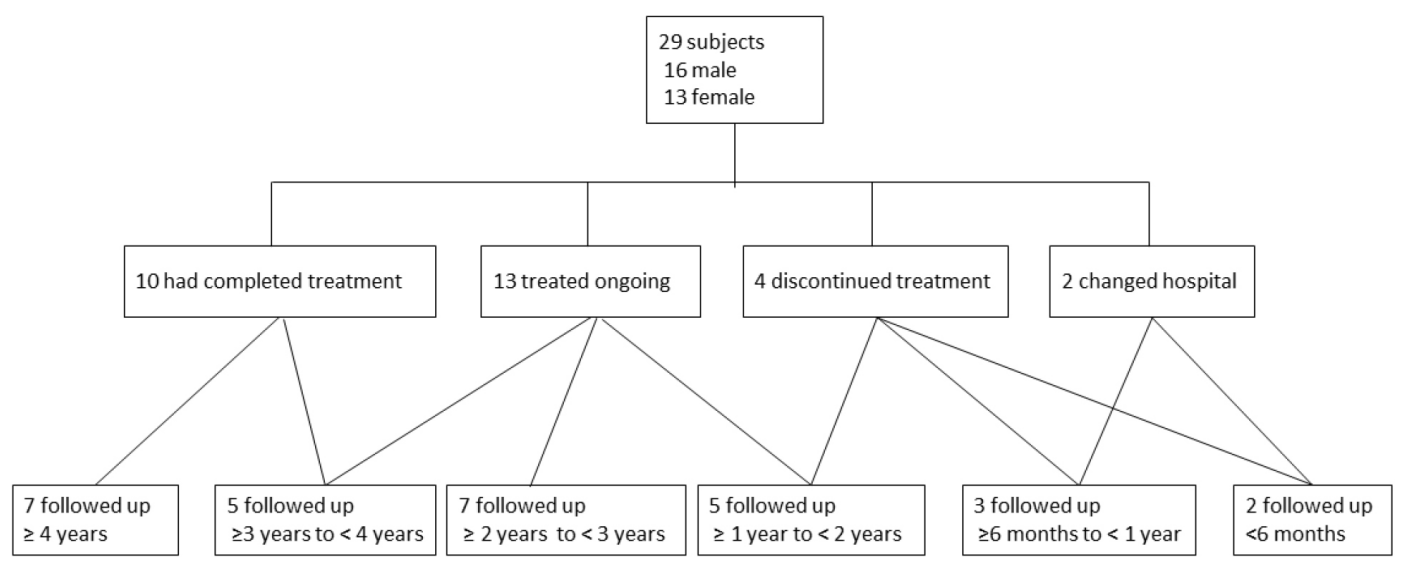

Figure 1. Profile of the patients in this study. 
Table 1. Clinical Features

\begin{tabular}{|c|c|}
\hline Parameters & n (\%) \\
\hline Total number & 29 \\
\hline \multirow[t]{2}{*}{ Sex } & Male: $16(55 \%)$ \\
\hline & Female: $13(45 \%)$ \\
\hline \multirow[t]{4}{*}{ Follow up status } & Completed: $10(34 \%)$ \\
\hline & Ongoing: 13 (45\%) \\
\hline & Discontinued: $4(14 \%)$ \\
\hline & Changed hospital: 2 (7\%) \\
\hline \multirow[t]{6}{*}{ Follow up duration } & $<6$ months: $2(7 \%)$ \\
\hline & $\geq 6$ months to $<1$ year: $3(11 \%)$ \\
\hline & $\geq 1$ year to < 2 years: $5(17 \%)$ \\
\hline & $\geq 2$ years to $<3$ years: $7(24 \%)$ \\
\hline & $\geq 3$ years to $<4$ years: $5(17 \%)$ \\
\hline & $\geq 4$ years: $7(24 \%)$ \\
\hline History of febrile seizure & $6(21 \%)$ \\
\hline Developmental disorders & $4(14 \%)$ (ADHD:2, ASD: 1, LD: 1) \\
\hline Todd paresis & $1(3 \%)$ \\
\hline History of family & $1(3 \%)$ \\
\hline Consanguinity & 0 \\
\hline \multirow[t]{2}{*}{ Seizure type } & Secondary Generalized:13 (45\%) \\
\hline & focal: $16(55 \%)$ \\
\hline \multirow[t]{3}{*}{ Focus of focal spikes } & Left: $14(34 \%)$ \\
\hline & Right: 9 (45\%) \\
\hline & Bilateral: $6(21 \%)$ \\
\hline
\end{tabular}

years, whereas another 7 [24\%] were followed up for $\geq 4$ years. At the initial seizure, 13 [45\%] patients had accompanying secondary generalization and just more than half had simple partial seizures of the facial or oral region. Six [21\%] patients had a medical history of febrile seizure, but none of the patients had a family history of epilepsy. Four [14\%] patients were complicated by developmental disorders, including attention deficit and hyperactivity disorder, a learning disorder, and autism spectrum disorder. At the initial seizure, Todd paralysis was found in one patient. None of the patients were the offspring of a consanguineous marriage. In the interictal EEG obtained during sleep, the focus was detected on the left in almost half of the patients, on the right in about a third, and on both sides in about one-fifth of the patients. Generalization on EEG was found in $14 \%$ of the patients.

Fifteen patients [55\%] were followed-up without treatment. Among the 14 [45\%] who were treated (Table 2), the mean period from disease onset to the start of the treatment was 7.1 months, and, on average, these children had about 3 seizures before the start of treatment. Administered drugs included carbamazepine (CBZ), zonisamide, levetiracetam (LEV), valproic acid, clonazepam, and lamotrigine. CBZ alone resulted in the disappearance of seizures in 5 patients, and $\geq 3$ drugs were used in 3 drug-re- sistant patients. CBZ was discontinued due to drugrelated eruption in 3 patients.

Twenty-four patients were followed-up for $\geq 1$ year and underwent EEG $\geq 2$ times during the intermittent period. Analysis of the correlation between age at seizure onset (months) and the duration of spike persistence (EEG abnormality) revealed a negative correlation (Spearman's $\rho-0.5140265 ; \mathrm{p}<0.01$; Fig. 2). Contrastingly, a positive correlation was found between the duration of EEG abnormality and the period until the disappearance of seizures (Spearman's $\rho$ 0.4900971; $<<0.05$; Fig. 3). However, no significant difference was found between age in months at the initial seizure and the period until the disappearance of seizures (Spearman's $\rho-0.3049274$; $\mathrm{p}>0.05$ ).

Among the 22 patients that did not have a follow up of $<1$ year, self-discontinuation of treatment or changing hospitals for treatment, 9 patients were in the NT group and 13 were in the TR group (Table 3 ). However, there were no significant differences in the age in months at seizure onset between the NT and TR groups. There were significant differences in the duration of EEG abnormality ( $\mathrm{p}<0.05)$, the period until the disappearance of seizures $(\mathrm{p}<0.01)$, and the number of seizures $(p<0.01)$ between the NT and TR groups. There was no significant difference in followup duration, sex, medical history of febrile seizures, 
Table 2. Features of BECTS Cases with or without Treatment

$\begin{array}{ll}\text { Parameters } & \mathrm{n}(\%) \\ \text { Total number } & 29 \\ \text { No treatment } & 16(52 \%) \\ \text { Treatment } & 13(45 \%) \\ \text { CBZ } & 13(92 \%) \\ \text { ZNS } & 4(28 \%) \\ \text { LEV } & 4(28 \%) \\ \text { VPA } & 3(21 \%) \\ \text { CZP } & 2(14 \%) \\ \text { LTG } & 2(14 \%) \\ \text { Monotherapy } & 7(50 \%) \\ \text { Two drug therapy } & 4(28 \%) \\ \text { Three drug therapy } & 2(14 \%) \\ \text { Four drug therapy } & 1(7 \%)\end{array}$

CBZ: Carbamazepine, ZNS: zonisamide, LEV: levetiracetam, VPA: valproic acid, CZP: clonazepam, LTG: lamotorigine

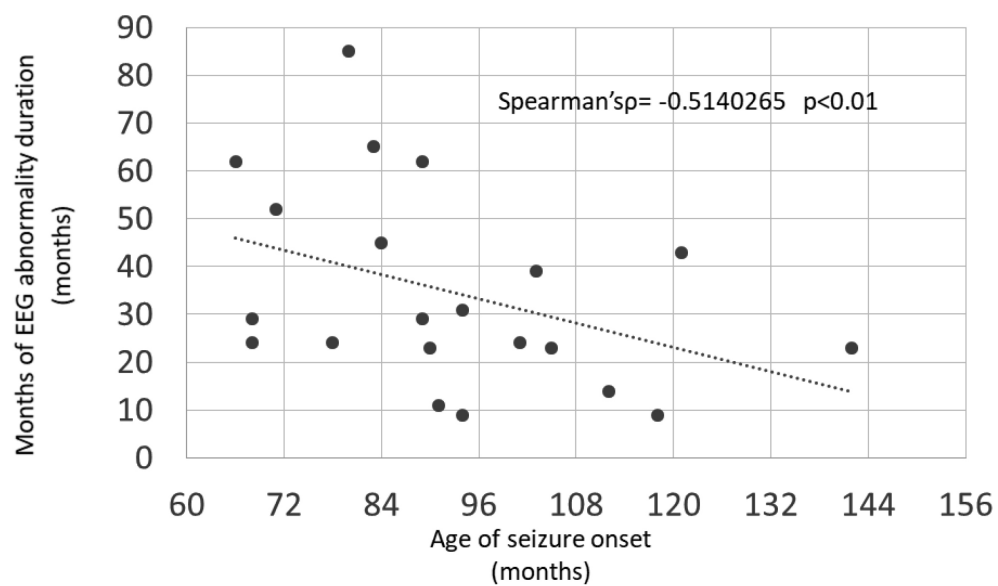

Figure 2. Scatterplot showing the negative correlation between months of spike persistence and age at seizure onset for all BECTS cases. (Spearman's $\rho-0.5140265, \mathrm{p}<0.01$ )

complication of developmental disorders, focus of the electroencephalogram, and electroencephalographic generalization. The odds ratio of treatment was 0.124 for seizure onset age ( $95 \%$ CI 0.017 to $0.90, \mathrm{p}<0.05$; Table 3). No significant difference was observed in the odds ratios of the other indices.

\section{Discussion}

In patients with BECTS, moderate doses of a single AED are usually sufficient ${ }^{5}$, but only, simple observation alone is needed in typical BECTS cases because the seizure frequency is low. Whether to treat BECTS is controversial. AEDs treatment is often not recommended, particularly if only focal seizures without impairment of consciousness occur and if the child and family are comfortable with this approach ${ }^{5}$. AEDs were found to significantly reduced generalized seizures but not partial seizures and after 4 to 14 years and more than 900 seizures, all patients were in remission without medication or injury6). Another meta-analysis concluded that early prediction of seizure outcome in a new patient with BECTS cannot be made with certainty ${ }^{7)}$. In the present study, we compared groups with $(n=9)$ and without treatment $(n=13)$. We found that the younger the age at seizure onset, the longer the duration of the EEG abnormality becomes, which agreed with a previous report ${ }^{8}$. Moreover, we observed a positive correlation be- 


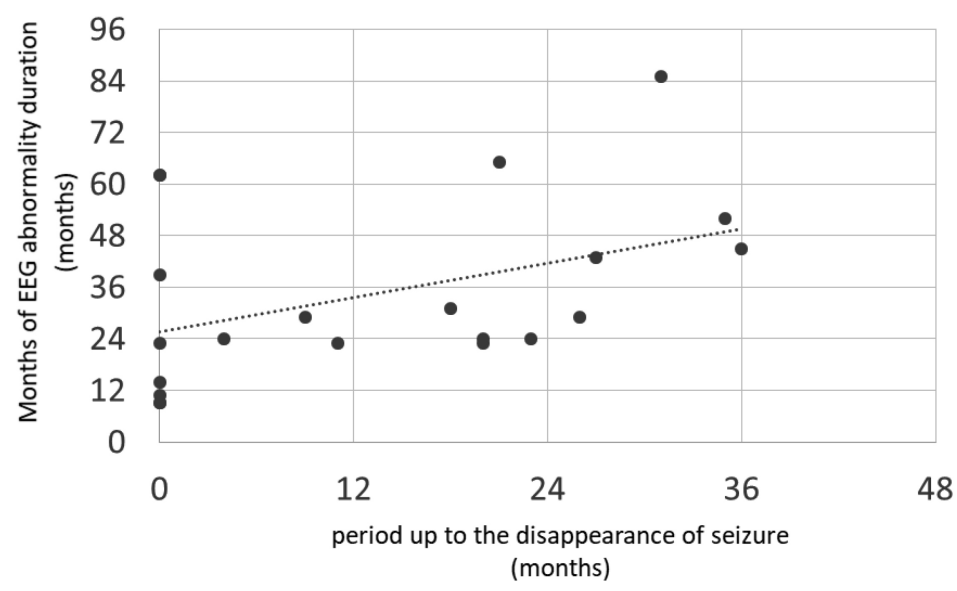

Figure 3. Scatterplot showing the positive correlation between months of spike persistence and the period until the disappearance of seizures for all BECTS cases. (Spearman's $\rho$ 0.4900971, p<0.05)

Table 3. Group Analysis of Treatment Intervention

\begin{tabular}{llll}
\hline & $\begin{array}{l}\text { No treated group } \\
\text { (NT) }\end{array}$ & Treated group (TR) & P value \\
\hline Number & 9 & 13 & \\
\hline Follow up duration (months) & $28.1 \pm 17.0$ & $47.4 \pm 25.9$ & $<0.05$ \\
\hline Age of seizure onset (years) & $8.2 \pm 1.6$ & $6.6 \pm 1.3$ & n.s \\
\hline $\begin{array}{l}\text { EEG abnormality duration } \\
\text { (months) }\end{array}$ & $23.8 \pm 16.1$ & $40.1 \pm 20.3$ & $<0.05$ \\
\hline Seizure persistence (months) & $3.0 \pm 5.7$ & $19.2 \pm 11.9$ & $<0.01$ \\
\hline Total number of seizures $(\mathrm{n})$ & $1.5 \pm 0.9$ & $3.3 \pm 0.82$ & $<0.05$ \\
\hline
\end{tabular}

tween the duration of EEG abnormality and the period up to the disappearance of seizures: the longer the duration of EEG abnormality becomes, the longer the duration of seizures may become.

Some reports showed that valproate and other AEDs were effective in focal epilepsies ${ }^{9-13)}$. However, Peters et al. ${ }^{6}$ reported that AEDs significantly reduced generalized seizures but did not reduce partial seizures. Various AEDs were used in the present study, and no patients had generalized seizure, but there were 3 patients with drug resistance. Recently, some studies suggested that the target of focal epilepsy treatment should be not only seizures but also quality of life ${ }^{14,15}$. Although, we could not investigate quality of life in this study, it will be necessary to follow up the patients in this study, especially the 3 patients with drug resistance.

Despite the benignity of BECTS, more evidence is being published about the involvement of cognitive functions ${ }^{16)}$. Operto et al. ${ }^{17)}$ reported that, LEV ther- apy in children with BECTS does not negatively affect the cognitive profile and that executive functions after 24 months were slightly improved. CBZ, valproic acid or LEV was recommended as the first choice on for BECTS cases in the Japanese epilepsy guideline ${ }^{18}$. Although we primarily used CBZ in this study, we should consider LEV use based on the result of the previous study ${ }^{17}$.

We found that, compared with the NT group, the TR group exhibited a longer duration of EEG abnormality. In addition, there was a significant difference between the groups in terms of the period until the disappearance of the seizures, although there was no significant difference in the age in months at seizure onset. These findings indicate that interventional treatment shortens the period to the disappearance of seizures and that the duration of the EEG abnormality is prolonged in TR cases. In fact, the purpose of treatment for BECTS is to ameliorate seizure, rather than to address the spikes on EEG per se. However, a re- 
Table 4. Multiple Logistic Regression Analysis

\begin{tabular}{llc}
\hline & Odds ratio & 95\% confidence intervals \\
Treatment & Reference & \\
Epilepsy onset age & $0.124^{*}$ & $0.017-0.910$ \\
Last follow up age & 4.632 & $0.613-35.014$ \\
History of febrile seizure & 1.068 & $0.048-23.943$ \\
Generalized/partial seizure & 0.315 & $0.019-5.159$ \\
complications due to developmental disorders & 0.247 & $0.020-3.018$ \\
Unilateral/Bilateral abnormal findings on EEG & 1.350 & $0.121-15.056$ \\
Generalization on interictal EEG abnnormality & 7.692 & $0.162-365.093$ \\
Duration of electroencephalographic abnormality & 1.003 & $0.950-1.058$ \\
$\mathrm{R}^{2}$ & 0.664 & \\
& & \\
\hline
\end{tabular}

cent study reported that interictal EEG abnormality is associated with cognitive dysfunction or neuropsychiatric disorder ${ }^{2,19,20)}$.

A previous study found that diffusing foci at the follow-up EEG and delayed treatment (duration $>1$ year) are the two main risk factors for drug-resistance in BECTS $^{21)}$. The multivariate analysis in the present study showed that only age at seizure onset was related to the odds ratio of treatment. We analyzed generalization or/not on interictal EEG only on the first examination. It's necessary that periodic follow-up of interictal EEG.

The limitations of this study included that it was a single-institution study with a small number of cases. In some patients, the follow-up period was not sufficiently long, and the mixture of cases, which may have included atypical benign partial epilepsy associated with BECTS, may have affected the results. The frequency of the appearance of paroxysmal discharges and dipoles was not studied. Therefore, it will be necessary to accumulate more data with larger sample sizes and to follow up the prognosis of these patients for a longer period of time.

The results of the present study suggested that there may be an association between interictal EEG abnormalities and the duration of seizure in patients with BECTS. Therefore, we concluded that abnormality on the interictal EEG may become a target of treatment in these patients.

\section{Conflicts of Interest}

The authors have nothing to disclose.

\section{References}

1) Parisi P, Paolino MC, Raucci U, et al. "Atypical forms" of benign epilepsy with centrotemporal spikes (BECTS): How to diagnose and guide these children. A practical/scientific approach. Epilepsy Behav 2017; 75: 165-169.

2) Hughes JR. Benign epilepsy of childhood with centrotemporal spikes (BECTS): To treat or not to treat, that is the question. Epilepsy Behav 2010; 19: 197-203.

3) Bektaş G, Tekin U, Yıldız EP, et al. Psychosocial and behavioral functioning and their relationship to seizure timing in children with benign epilepsy with centrotemporal spikes. Brain Dev 2017; 39: 515-520.

4) Tenney JR, Glauser T, Altaye M, et al. Longitudinal stability of interictal spikes in benign epilepsy with centrotemporal spikes. Epilepsia 2016; 57: 805-811.

5) Benhadis SR. Benign focal epilepsies of childhood. Post TW (ed), UpToDate, Waltham, MA. https://www.uptodate.com/contents/benignfocal-epilepsies-of-childhood. Retrieved on September 27, 2019.

6) Peters JM, Camfield CS, Camfield PR. Population study of benign rolandic epilepsy: is treatment needed?. Neurology 2001; 57: 537-539.

7) Bouma PA, Bovenkerk AC, Westendorp RG, et al. The course of benign partial epilepsy of childhood with centrotemporal spikes: a metaanalysis. Neurology 1997; 48: 430-437.

8) Kim H, Kim SY, Lim BC, et al. Spike persistence and normalization in benign epilepsy with centrotemporal spikes-implications for management. Brain Dev 2018; 40: 693-698.

9) Ma CK, Chan KY. Benign childhood epilepsy with centrotemporal spikes: a study of 50 Chinese children. Brain Dev 2003; 25: 390-395.

10) Tzitiridou M, Panou T, Ramantani G, et al. Oxcarbazepine monotherapy in benign childhood epilepsy with centrotemporal spikes: a clinical 
and cognitive evaluation. Epilepsy Behav 2005; 7: 458-467.

11) Bello-Espinosa LE, Roberts SL. Levetiracetam for benign epilepsy of childhood with centrotemporal spikes-three cases. Seizure 2003; 12: 157-159.

12) Coppola G, Franzoni E, Verrotti A et al. Levetiracetam or oxcarbazepine as monotherapy in newly diagnosed benign epilepsy of childhood with centrotemporal spikes (BECTS): an openlabel, parallel group trial. Brain Dev 2007; 29: 281-284.

13) Hughes JR. Benign epilepsy of childhood with centrotemporal spikes (BECTS): to treat or not to treat, that is the question. Epilepsy Behav 2010; 19: 197-203.

14) Taskiran E, Matur Z, Gül G, et al. The Impact of Affective State on Quality of Life in Focal Epilepsy in Turkey. J Neurosci Rural Pract 2019; 10: 267-272.

15) Baranowski CJ. The Quality of Life of Older Adults With Epilepsy: A Systematic Review Seizure 2018; 60: 190-197.

16) Tristano I, Nicita F, Garone G, et al. Could Ro- landic spikes be a prognostic factor of the neurocognitive outcome of children with BECTS? Epilepsy Behav 2018; 86: 157-162.

17) Operto FF, Pastorino GMG, Mazza R, et al. Cognitive profile in BECTS treated with levetiracetam: a 2-year follow-up. Epilepsy Behav 2019; 97: 187-191.

18) Tenkan Shinryou Guideline Sakusei-Iinkai (eds), Tenkan Shinryo Guideline [In Japanese], 1st Edition, Igaku-shoin, Tokyo, 2018: 65-66.

19) Bektaş G, Tekin U, Yıldız EP, et al. Psychosocial and behavioral functioning and their relationship to seizure timing in children with centrotemporal spikes. Brain Dev 2017; 39: 515520.

20) Garcia-Ramos C, Jackson DC, Lin JJ, et al. Cognition and brain development in children with benign epilepsy with centrotemporal spikes. Epilepsia 2015; 56: 1615-1622.

21) Xiao F, An D, Chen S, et al. Clinical and Electroencephalographic (EEG) Features Associated With Refractoriness in Benign Childhood Epilepsy With Centrotemporal Spikes. J Child Neurol 2015; 30: 1591-1597. 